\title{
HIGH SOLUBLE ENDOGLIN LEVELS AFFECT BILE ACIDS AND CHOLESTEROL METABOLISM IN MICE LIVER
}

\section{Objectives} so far and bile acids turnover in liver.

\section{Methods} expression analyses.

\section{Results} transporter.

.

Plast

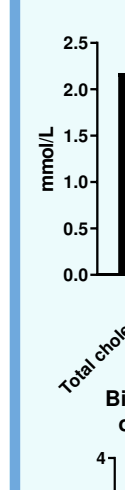
Eva Dolezelova ${ }^{1}$, Alena Prasnicka ${ }^{1}$, Ivone Cristina Igreja e Sá ${ }^{1}$, Milos Hroch $^{3}$, Radomir Hyspler ${ }^{4}$, Alena Ticha $^{4}$, Miguel Pericacho ${ }^{5}$, Stanislav Micuda ${ }^{2}$, Petr Nachtigal ${ }^{1}$
${ }^{1}$ Department of Biological and Medical Sciences, Faculty of Pharmacy in Hradec Kralove, ${ }^{2}$ Department of Pharmacology,
Department of Biochemistry, Faculty of Medicine in Hradec Kralove, Charles University, Czech Republic; ${ }^{4}$ Department of Research and Development, University Hospital Hradec Kralove, Czech Republic; ${ }^{5}$ Department of Physiology and Pharmacology, University of Salamanca, Salamanca, Spain
Endoglin (CD 105) is transmembrane glycoprotein that acts as receptor for TGF- $\beta$ and modulates cellular responses to this pleiotropic cytokine. It's soluble form (sEng) is a plasma protein released by the cleavage of the extracellular domain of endoglin by membrane-type metalloproteinase 1.
Plasma levels of sEng are increased in cardiovascular and metabolic diseases including hypercholesterolemia, atherosclerosis, and type II diabetes mellitus. These diseases also affect liver functions and metabolism. However, sEng direct effects on cholesterol metabolism in liver have not been published
Therefore, we hypothesized that high levels of sEng will affect cholesterol
- Six-month-old transgenic male mice overexpressing human sEng on CBAxC57BL/6J background (sEng) and wild type mice (WT) underwent in vivo study with bile collection for $45 \mathrm{~min}$, with subsequent biochemical and
- Cholesterol, bile acids, and liver tests were determined in plasma. Spectra of bile acids in the bile and stool were evaluated by LC-MS/MS.
- Expressions of enzymes and transporters responsible for cholesterol and bile acids homeostasis in liver and ileum were assessed by qRT-PCR and Western blot. We focused to molecules mediating cholesterol uptake into hepatocytes (Sr-b1, Ldlr), synthesis of cholesterol (Hmgcr), cholesterol esterification (Acat2), biliary cholesterol and phosphatidylcholine efflux (Abcg5/8, Mdr2, respectively), and plasma efflux of cholesterol (Abca1). Also were analyzed molecules enabling bile acids uptake from plasma into hepatocytes (Ntcp, Oatp2), efflux of bile acids into bile (Bsep, Mrp2), efflux of bile acids back to plasma (Mrp3, Mrp4), bile acids synthesis (Cyp7a1, the rate-limiting enzyme in the catabolism of cholesterol into bile acids, Cyp8b1 and Cyp27a1, enzymes of alternative pathway of BA synthesis). To describe the complex BA enterohepatic circulation, expression of Asbt for $B A$ uptake and Ost $\alpha / \beta$ and Fgf15 for efflux of BA into portal blood.

\section{High levels of $s$ Eng decreased plasma cholesterol concentration}
Transgenic mice with increased sEng demonstrated decreased plasma total and LDL cholesterol levels. This effect paralleled upregulation of hepatic Sr-b1 and Ldlr proteins responsible for cholesterol uptake into hepatocytes and increased Abcg8 protein level for cholesterol efflux into bile. Unchanged protein expression of Hmgcr and fractional cholesterol synthesis rate supported increased uptake of cholesterol from blood as the main mechanism of increased liver and reduced plasma cholesterol concentrations. Biliary excretion of phospholipids was increased despite the unchanged expression of their Mdr2
20 